\title{
Wirkungsforschung ist machbar
}

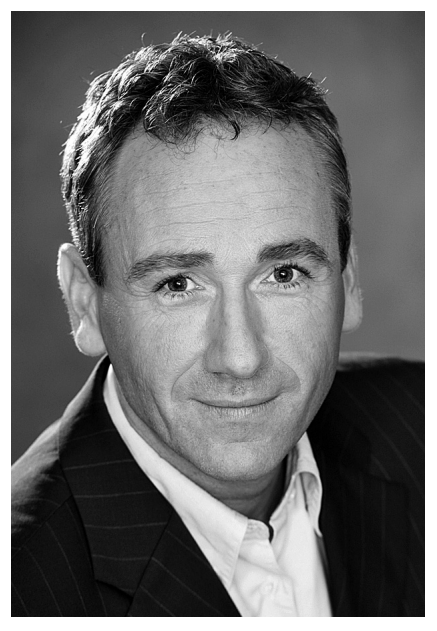

VON MICHAEL MACSENAERE Prof. Dr. Michael Macsenaere ist Geschäftsführender Direktor des Instituts für Kinder- und Jugendhilfe gGmbH in Mainz. Seine Forschungsschwerpunkte sind Evaluation im Sozialwesen, Qualitätsentwicklung und wirkungsorientierte Steuerung, ressourcenorientierte Pädagogik und Sportpsychologie.

macsenaere@ikj-mainz.de

\author{
Wirkungsforschung in der Sozialen Arbeit ist eine \\ noch junge Disziplin. Dennoch haben sich bereits \\ zahlreiche Standards und Methoden herausgebildet, \\ wie ein Blick in die Kinder- und Jugendhilfe \\ zeigt. Die dort gewonnenen Verfahren und \\ Empfehlungen sind grundsätzlich auch auf andere \\ Tätigkeitsfelder der Sozialen Arbeit übertragbar.
}

Erziehungshilfen nach dem Kinderund Jugendhilfegesetz $(\mathbb{S} 27$ ff. SGB VIII) wurden 2011 in Deutschland von knapp einer Million junger Menschen in Anspruch genommen und stellen somit eine bedeutende Größe in der Sozialwirtschaft dar.

In Anbetracht der Gesamtkosten von 7,1 Milliarden Euro (Schilling, 2013) scheint es nahezuliegen, die Ergebnisse aus mittlerweile zwanzig Jahren Wirkungsforschung bevorzugt zur Legitimation dieses Praxisfeldes zu nutzen. Im Übrigen fordert auch der 14. Kinder- und Jugendbericht von den öffentlichen und freien Trägern »die fachliche Wirksamkeit und ihr Kosten-Nutzen-Verhältnis auszuweisen und die Wirkungen ihrer Leistungen systematisch zu evaluieren « (BMFSFJ, 2013).

Die Befunde aus wirkungsorientierten Evaluationen allein zur Legitimation $\mathrm{zu}$ verwenden würde allerdings nur einen kleinen Teil des Nutzensspektrums von Wirkungsforschung abdecken und sollte durch weitere Aspekte ergänzt werden.

\section{Legitimation}

Aber kann die Wirkungsforschung überhaupt einen Beitrag zur Legitimation der Erziehungshilfen liefern? Und wenn ja, welchen? Ein Überblick über die rund einhundert vorliegenden deutschsprachigen Wirkungsstudien belegt diesem Bereich hohe Erfolgsquoten, die je nach
Studie und untersuchter Hilfe zwischen 60 und 90 Prozent liegen, wie auch merkliche positive Effektstärken. Darüber hinaus zeigen erste Analysen, dass die Kosten-Nutzen-Relation von Heimerziehung 1:3 beträgt (Roos, 2005). Ein Euro, der heute investiert wird, führt für die Gesellschaft demnach zu langfristigen volkswirtschaftlichen Nutzeneffekten von drei Euro.

Diese Ergebnisse attestieren der Erziehungshilfe also eine ausgeprägte Effektivität und Effizienz und somit Sinnhaftigkeit. Natürlich lassen sich solche Aussagen von dieser hoch aggregierten Ebene auf Einrichtungsebene herunterbrechen und von Trägern und Einrichtungsleitungen zur Legitimation der eigenen Arbeit verwenden. So nutzen seit 1999 über 200 Einrichtungen beispielsweise die »Evaluation Erzieherischer Hilfen (EVAS) « (Macsenaere \& Knab, 2004), um die Effekte ihrer Arbeit wissenschaftlich abgesichert zu erheben und sich damit einen Argumentationsvorsprung zu verschaffen.

\section{Sechs Wirkfaktoren}

Eine solche Nutzung von Wirkungsforschung ist zwar durchaus legitim, sollte aber nicht den Blick auf das ihr innewohnende Potenzial zur Qualitätsentwicklung verstellen. So werden in den meisten Wirkungsstudien nicht nur die Effekte sozialer Arbeit untersucht, sondern auch die zugrundeliegenden Wirkfaktoren. $\rightarrow$ 


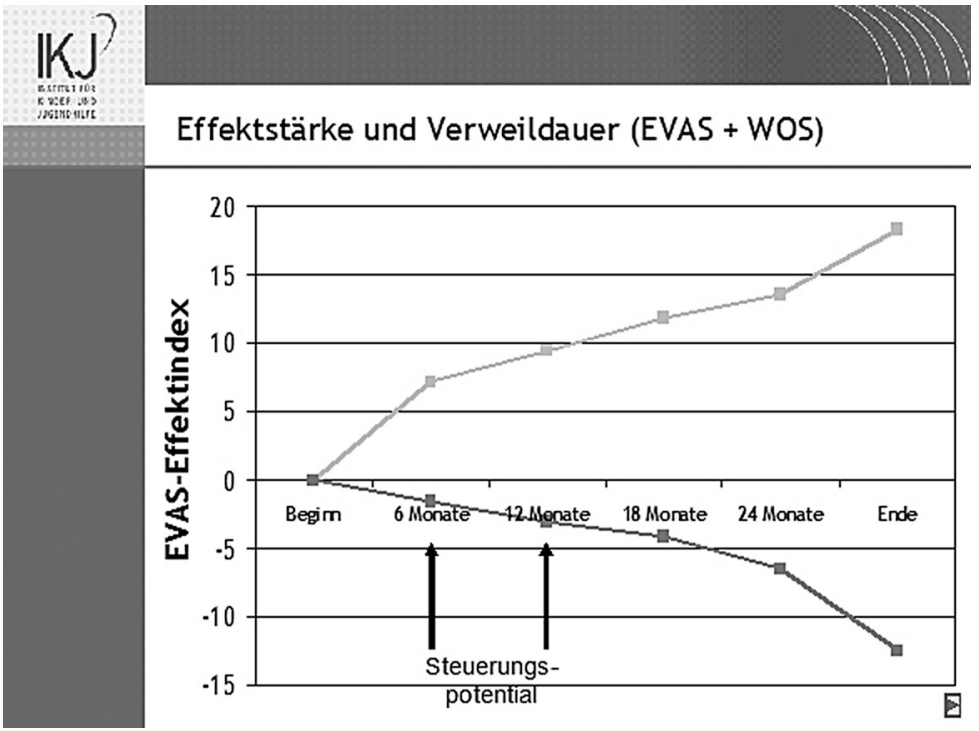

Schon nach einem halben Jahr einer Erziehungshilfe kann mit einer hohen Zuverlässigkeit der Erfolg oder der Misserfolg einer Maßnahme prognostiziert werden. Im Falle eines positiven Effekte-Index kann dann der begonnene Weg stabil weitergeführt werden (obere Kurve). Im Falle eines negativen Effekte-Index und der daraus resultierenden negativen Prognose besteht hingegen sofortiger Handlungsbedarf (untere Kurve).

Die zentralen dieser empirisch nachgewiesenen Faktoren sollten zukünftig konsequenter und nachhaltiger in den Fokus von Jugendämtern und Leistungserbringern gerückt werden, um passgenauere, effektivere und effizientere Hilfen zu formen. Die nachfolgenden Faktoren haben sich hierfür als besonders wirkmächtig erwiesen (Macsenaere \& Esser, 2012):

1. Kooperation: Als zentraler Wirk- faktor pädagogischer Arbeit zeigt sich die Kooperation von Eltern und jungem Menschen. Gelingt diese aktive Mitarbeit im Rahmen der Hilfe, verbessert sich die Aussicht auf Erfolg erheblich - unterbleibt sie, wird ein Misserfolg der Hilfe wahrscheinlich. Eine notwendige, aber nicht hinreichende Voraussetzung für Kooperation stellt die Partizipation von Eltern und jungem Menschen dar, die jedoch ergänzt werden muss um das stetige Bemühen, eine gelingende Hilfe zur Selbsthilfe zu schaffen.

2 Vermeidung von Jugendhilfekarrieren: Je mehr Hilfen in Anspruch genommen wurden, desto höher ist die "Änderungsresistenz« des jungen Menschen, desto geringer ist also die zu erwartende Effektivität der Maßnahme oder Intervention. Es gilt daher die durchaus üblichen Jugendhilfekarrieren zu vermeiden, indem frühzeitig eine adäquate Hilfe gewährleistet wird.
3 Sozialpädagogische Diagnostik: 3. In Hinblick auf die Arbeit im Jugendamt erweist sich eine einheitliche und systematisierte sozialpädagogische Diagnostik als sinnvoll, wie sie beispielsweise mit den bayerischen Sozialpädagogischen Diagnose-Tabellen vorliegt (Macsenaere et al., 2009). Damit wird die Zuweisungsqualität des Jugendamtes erhöht, was in der Folge zu effektiveren Hilfen führt. Das Nutzen einer einheitlichen Fachsprache ist zudem eine Grundlage zunehmender Transparenz.

1 Indikation: Eine zentrale Aufga4. be des Jugendamtes ist die sogenannte Zuweisungsqualität, also eine geeignete oder indizierte Hilfe auszuwählen. Effektestudien (EVAS, EST!, JES) zeigen, dass es den Fachkräften des Allgemeinen Sozialen Dienstes in immerhin der Hälfte der Fälle gelingt, die am ehesten geeignete Hilfe zu wählen. Dem stehen aber circa 30 Prozent der Fälle gegenüber, bei denen eine nicht geeignete Hilfe gewählt wird, teilweise auch aus Kostengründen. Hier besteht in den nächsten Jahren noch Qualitätsentwicklungsbedarf, um die vorliegenden wissenschaftlichen Erkenntnisse für die Praxis der Jugendämter nutzbar zu machen: So können auf der Grundlage von über 40.000 evaluierten Hilfeverläufen mittlerweile mit einer hohen Prognosegüte Empfeh- lungen gemacht werden, welche Hilfe in der jeweils vorliegenden Ausgangskonstellation in welchem Ausmaß geeignet oder nicht geeignet ist.

5. Mitarbeiterqualifikation: Wird 2. hier ein Minimalstandard unterschritten, beispielsweise keine (sozial-) pädagogische Ausbildung und Erfahrung, steigt die Wahrscheinlichkeit für teilweise drastische Misserfolge an.

6. Ressourcenorientierung: Eine stärkere Ressourcenorientierung im Jugendamt und in den Einrichtungen begünstigt die Effektivität wie die Nachhaltigkeit der erreichten Effekte. Die Hilfen, bei denen zumindest ein Hilfeplanziel die Förderung einer Ressource anstrebt, erreichen stärkere Effekte als Hilfen mit rein defizitorientierten Hilfeplanzielen.

\section{Wirkungsorientierte Steuerung}

Jenseits des Erkennens dieser Wirkfaktoren lassen sich die Ergebnisse evidenzbasierter Evaluationen zumindest in zweifacher Hinsicht für eine wirkungsorientierte Steuerung verwenden: auf Einzelfallebene und auf aggregierten Ebenen.

Auf Einzelfallebene findet zunehmend ein aktuelles Ergebnis aus der Evaluation erzieherischer Hilfen (IKJ, 2008) Eingang in die Praxis: Schon nach einem halben Jahr ist es mit einer hohen Zuverlässigkeit möglich, den späteren Erfolg oder Misserfolg der Hilfe zu prognostizieren. Fallverläufe mit einem positiven Effektindex entwickeln sich in der Regel auch in der Folge weiter positiv. Umgekehrt sind negative Effektwerte schon nach sechs Monaten sehr kritisch zu bewerten und sollten eine Reflexion und gegebenenfalls Umsteuerung nach sich ziehen, um einer weiteren sukzessiven Verschlechterung entgegenzuwirken (vgl. Abbildung).

Dieses Wissen nutzen zunehmend Einrichtungen und Jugendämter für ihre Hilfeplangespräche zur kritischen Reflexion des bisherigen Verlaufs. Im Falle eines positiven Effekte-Index kann dann der begonnene Weg stabil weitergeführt werden (obere Kurve). Im Falle eines negativen Effekte-Index und der daraus resultierenden negativen Prognose besteht hingegen sofortiger Handlungsbedarf (untere Kurve). 
Einrichtungen und Jugendämter, die mit diesem prognostischen Frühwarnsystem arbeiten, können damit Fehlentwicklungen frühzeitig erkennen und umgehend korrigieren.

Auf aggregierten Ebenen sind differenzierte Aussagen zu Hilfearten, Bezirken, Einrichtungsgruppen, Klientelmerkmalen (Geschlecht, Nationalität, Alter usw.) etc. möglich. Zudem lassen sich Einrichtungsgruppen in einem internen Benchmarking miteinander vergleichen. Auf Organisationsebene ermöglichen wirkungsorientierte Evaluationen der Einrichtung oder dem Jugendamt einen Überblick zu Klientel, Hilfearten, Hilfedauer, Kosten, Erfolgen, Effizienz etc. Darüber hinaus ist es möglich, die eigenen Ergebnisse mit denen anderer Einrichtungen zu vergleichen und dadurch eine Stärken-Schwächen-Analyse zu erhalten. Auf überregionaler und jugendhilfepolitischer Ebene lassen sich Modelle gelingender, aber auch misslingender Praxis identifizieren und für Optimierungsprozesse nutzen.

Die beiden dargestellten Steuerungsoptionen sind unbedingt von einer wirkungsorientierten Steuerung im Sinne einer Bewertung der Leistungserbrin- ger durch das Jugendamt zu unterscheiden, die sich mit rein materiellen Anreizstrukturen(z. B. Bonus-MalusSystem) kombiniert als nicht zielführend erwiesen hat (ISA, 2009).

\section{Empfehlungen}

Mit welchen Methoden kann eine zuverlässige und trotzdem leistbare Wirkungsmessung in der Praxis erfolgen? Zur Beantwortung dieser oft gestellten Frage kann auf die Erfahrung aus mittlerweile zwanzig Jahren Wirkungsmessung zurückgegriffen werden, aus denen folgende Empfehlungen abgeleitet werden können:

- Erfassung intendierter und nicht intendierter Wirkungen: Jede Hilfe erzielt nicht nur beabsichtigte (intendierte) Wirkungen, sondern auch Nebenwirkungen (nicht intendierte Wirkungen), die erheblichen Einfluss auf den Erfolg und Misserfolg der Intervention ausüben und berücksichtigt werden müssen.

- Prospektives Längsschnittdesign: Zuverlässige Wirkungsmessung muss hilfebegleitend (prospektiv) und mit mindestens zwei Erhebungszeitpunkten (Anfang und Ende der Hilfe) erfolgen. Falls die Ergebnisse der Evaluation auch zur Steuerung verwendet werden sollen, müssen weitere Erhebungszeitpunkte (z. B. halbjährlich) eingeplant werden.

- Wirkungsindikatoren: Zumeist werden Veränderungen der Ressourcen und Defizite des jungen Menschen und des Umfelds gemessen. Aktuell gewinnt der Capabilitiy Approach nach Nussbaum und Sen (1993) bei der Operationalisierung von Wirkung zunehmend an Bedeutung. Gemeint sind damit die Grundbefähigungen eines Menschen, die nicht nur von den eigenen Fähigkeiten, sondern auch von den gesellschaftlichen Rahmenbedingungen abhängig sind.

- Berücksichtigung sämtlicher relevanter Sichtweisen: Umfassende Wirkungsstudien erfassen drei Wirkungsdimensionen: 1. Effect - direkt ersichtliche und objektiv nachweisbare Interventionswirkung, die durch Fachkräfte oder Experten erfasst wird; 2. Impact - Subjektive Wirkung beim Hilfeadressaten (junger Mensch

\section{Was man unter Wirkungsanalyse versteht}

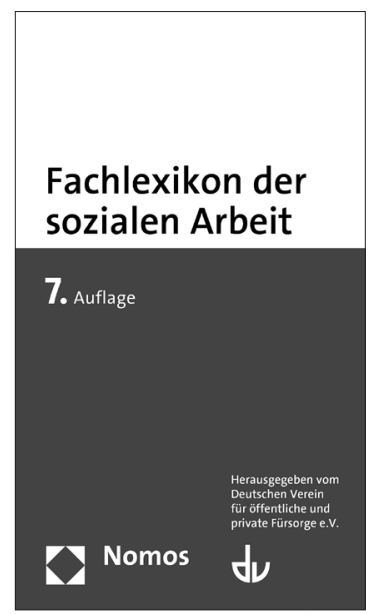

Wirkungsanalyse bezeichnet die Untersuchung eines ursächlichen (kausalen) Zusammenhangs zwischen einer Maßnahme (Intervention, Programm) und einer mit der Maßnahme in Verbindung stehenden Zielgröße. Die Wirkungsanalyse betrachtet eine Beobachtungseinheit (z. B. Person), die an einer Maßnahme (z. B. Fortbildungsprogramm, Medikamenteneinnahme) teilnehmen bzw. einer Intervention ausgesetzt sein kann oder nicht. Von Interesse ist der Effekt auf eine nach der Maßnahme gemessene
Zielgröße (z. B. Beschäftigungswahrscheinlichkeit, Blutdruck). Die Zielgröße kann für jede Person zwei potenzielle Ergebnisse annehmen: eines im Teilnahmefall und eines im Nichtteilnahmefall. Die Differenz aus beiden misst den kausalen Effekt der Maßnahme. Da für die einzelne Beobachtungseinheit nur einer der Fälle tatsächlich eintreten kann, ist das jeweils andere potenzielle Ergebnis eine nicht in Daten beobachtbare kontrafaktische Größe: »Was wäre mit der teilnehmenden Person (hinsichtlich der Zielgröße) geschehen, wenn sie nicht teilgenommen hätte?" Die Beantwortung dieser Frage ist Kern der Wirkungsanalyse. Hierzu verwendet die empirische Wirkungsanalyse in der Regel Mittelwerte und vergleicht das durchschnittliche Ergebnis der Teilnehmenden mit dem durchschnittlichen Ergebnis einer Vergleichs- oder Kontrollgruppe. Die Vergleichssituation ist begrifflich weit gefasst und kann beispielsweise auch durch den vor der Maßnahme ermittelten Wert der Zielgröße der Teilnahmegruppe abgebildet werden: Ein derartiger Vorher-NachherVergleich ist eine Möglichkeit, die kontrafaktische Frage zu beantworten. Andere Methoden sind beispielsweise der Querschnittsvergleich oder Matching (Vergleich mit "statistischen Zwillingen«). Jeder Methode liegt eine Identifikationsannahme zur Beantwortung der kontrafaktischen Frage - d. h. der Auswahl der adäquaten Vergleichsdimension - zugrunde, deren Plausibilität abhängig vom Maßnahmenkontext ist. Als methodisch stichhaltigste Vorgehensweise zur Wirkungsanalyse gilt das randomisierte Experiment, also die zufällige Zuordnung aller potenziellen Teilnehmenden in Teilnahme- und Kontrollgruppe. Dies ist in der medizinischen Forschung üblich und wird beispielsweise in den USA auch zur Wirkungsanalyse arbeitsmarktpolitischer Programme verwendet. Ein Experiment gewährleistet die Vergleichbarkeit beider Gruppen in allen relevanten beobachtbaren (z. B. Alter, Geschlecht, Ausbildungsstand) und unbeobachtbaren (z. B. Motivation, Begabung) Faktoren. Nicht-experimentelle Ansätze können in der Regel unbeobachtbare Charakteristika nicht berücksichtigen.

\section{Jochen Kluve}

Quelle: Deutscher Verein für öffentliche und private Fürsorge e. V. (Hg.): Fachlexikon der sozialen Arbeit. 7. Auflage. Nomos Verlagsgesellschaft, Baden-Baden 2011. 1.139 Seiten. 44,- Euro. ISBN 978-3-8329-5153-5. 
oder Familie); 3. Outcome - Mittelbare Wirkung auf den Sozialraum und die Gesellschaft, beispielsweise Verringerung der Kriminalität und volkswirtschaftliche Effekte.

- Aggregationsebenen: Erhebungen müssen einzelfallbezogen erfolgen, können aber auf verschiedenen Ebenen (Einzelfall, Gruppe, Institution, Region etc.) aggregiert ausgewertet werden.

- Über den Tellerrand blicken: Institutionsübergreifende und überregionale Untersuchungen ermöglichen Vergleiche (z. B. Stärken-SchwächenAnalysen) und ein gemeinsames Voneinander-Lernen.

benen wissenschaftlichen Standards hinsichtlich der Alltagstauglichkeit und Flexibilität erhebliche Fortschritte erzielen.

Ebenfalls interessant ist es, Kennziffern zur pädagogischen Wirksamkeit mit betriebswirtschaftlichen Kennziffern zu kombinieren. Dieser interdisziplinäre und innovative Weg wird durch das »Jugendhilfe-Cockpit« ermöglicht, mit dem erstmals die Produktivität, Wettbewerbsstärke, Prozessqualität und Wirksamkeit einer Institution sichtbar gemacht und zueinander in Bezug gesetzt werden (www.jugendhilfe-cockpit. de; Macsenaere,2012).

\section{"Studienergebnisse zur Wirkung von Erziehungshilfen können durchaus auf Einrichtungsebene herunter gebrochen werden"}

- Berücksichtigung der Evaluationsstandards: Nach der Gesellschaft für Evaluation (Degeval, 2008) sollten Evaluationen nützlich, durchführbar, fair und genau sein (ausführliche Beschreibung der Standards unter www. degeval.de). Darunter fallen die klassischen Testgütekriterien Objektivität (Unabhängigkeit der Ergebnisse des Verfahrens vom Anwender), Reliabilität (Zuverlässigkeit und Genauigkeit der Messung) sowie Validität (Gültigkeit: Wird das gemessen, was gemessen werden soll oder etwas anderes).

- Alltagstauglichkeit beachten, prüfen und sicherstellen: Die Forderung des 14. Kinder- und Jugendberichts, dass öffentliche und freie Träger die fachliche Wirksamkeit ihrer Leistungen nachweisen sollten, kann nur dann umgesetzt werden, wenn das Verfahren zum Wirkungsmessen in hohem $\mathrm{Maße}$ alltagstauglich ist. Dies betrifft sowohl den Umfang und den damit verbundenen Zeitaufwand wie die fachliche und technische Integration in den jeweiligen Workflow. Dank der Mitarbeit von Leistungserbringern und Jugendämtern wird das aktuell entwickelte Programm »Evaluation Erzieherischer Hilfen (EVAS 5.0)« trotz Gewährleistung der beschrie-

\section{Literatur}

Bundesministerium für Familie, Senioren, Frauen und Jugend (2013). 14. Kinder- und Jugendbericht. Bericht über die Lebenssituation junger Menschen und die Bestrebungen und Leistungen der Kinder- und Jugendhilfe in Deutschland.

DeGEval - Gesellschaft für Evaluation e. V. (Hg.) (2008): Standards für Evaluation, 4. Unveränderte Auflage. Mainz. ISA Planung und Entwicklung GmbH \& Universität Bielefeld (2009): Praxishilfe zur wirkungsorientierten Qualifizierung der Hilfen zur Erziehung. Münster: ISA.

IKJ Institut für Kinder- und Jugendhilfe (2008). EVAS-Highlights 2007: Wirkungsorientierte Steuerung im Einzelfall. Mainz: Institut für Kinder- und Jugendhilfe.

Jugendhilfe-Cockpit:

http://www.jugendhilfe-cockpit.de/

Macsenaere, M. \& Esser, K. (2012). Was wirkt in der Erziehungshilfe? München: Reinhardt. Macsenaere, M. \& Knab, E. (2004): EVAS - Eine Einführung. Freiburg: Lambertus. Macsenaere, M. Paries, G. \& Arnold, J. (2009). EST! Evaluation der Sozialpädagogischen Diagnose-Tabellen - Abschlussbericht. München: Bayerisches Staatsministerium für Arbeit und Sozialordnung, Familie und Frauen. Macsenaere, M. (2012). Wirkungscontrolling in der Jugendhilfe: das Jugendhilfe-Cockpit. In Bundesarbeitsgemeinschaft der Freien Wohlfahrtspflege (BAGFW) (Hg.): Den Wandel steuern. Personal und Finanzen als Erfolgsfaktoren (S. 307-312). Baden-Baden: Nomos. Nussbaum, M. \& Sen, A. (Hg.) (1993).

The Quality of Life. Oxford: Clarendon Press. Roos, K. (2005): Kosten-Nutzen-Analyse von Jugendhilfemaßnahmen. In: Petermann, F. (Hg.): Studien zur Jugend- und Familienforschung. Band 23. Frankfurt am Main: Lang. Schilling, M. (2013). Ausgabenentwicklung in der Kinder- und Jugendhilfe. Vortrag auf der Sitzung des BVkE-Vorstandes am 6.2.2013 in Köln. 